\title{
UNIVERSAL SCALING FUNCTION FOR FEL GAIN*
}

\author{
L.H. Yu and S. Krinsky \\ National Synchrotron Light Source \\ Brookhaven National Laboratory \\ Upton, New York 11973 USA \\ R.L. Gluckstern \\ Department of Physics and Astronomy \\ University of Maryland \\ College Park, Maryland 20743 USA
}

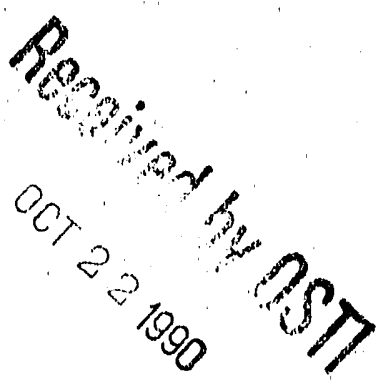

\section{$\underline{\text { Abstract }}$}

We have developed an analytic calculation of FEL gain in the exponential regime taking into account the finite emittance, energy spread, focusing and betatron oscillation of the electron beam, and the diffraction and guiding of the radiation. The gain is expressed in terms of a universal scaling function with only three independent parameters. Excellent agreement is found with results of numerical simulation.

*This work was performed under the auspices of the U.S. Department of Energy.

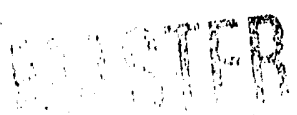

1

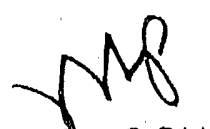


We have recently developed an analytic calculation [1] of the FEL gain valid in the regime of exponential growth before saturation, based upon a dispersion relation $[2,3]$ derived from the Vlasov-Maxwell eguations. Our treatment includes all of the effects of energy spread, emittance, and focusing of the electrun beam, and the diffraction and guiding of the radiation. The dispersion relation is solved using a variational approximation [4]. With a reasonable choice of frequency detuning yielding near maximum gain, we have shown that the e-folding length is a universal function of three dimensionless variables (emittance to wavelength ratio, scaled electron focusing strength, and scaled electron energy spread), and a dimensionless parameter measuring transverse current. The importance of dimensionless scaling variables was discussed in refs. [5] and [6]. Our scaling relations [1] are new results, and we have presented the first calculation of the universal scaling function determining FEL gain. Some graphs of the universal function are given in Figs. 1a,b,c. These graphs can be used for quick estimates of the gain for quite general parameters.

Until now, only large simulation codes $[7,8,9]$ requiring long CPU running times on fast computers could inciude all the effects determining FEL gain. Our analytic approach has several advantages: rapid computation time, elucidation of the dependence of the gain on the large number of system parameters, and the ease of design optimization. We have checked that the variational approximation yields agreement to within $1 \%$ with exact results [10] which we have derived for a parallel electron beam with finite beam size and energy spiand. In this note, we present a comparison of our analytic results with several cases with nonvanishing emittance run on the simulation codes FELEX and TDA. Agreement of the calculated gain length is found to within a few percent. 
In our calculation, the electron beam's energy distribution $h(\gamma)$ is Gaussian, with average energy $\gamma_{\mathrm{o}} \mathrm{mc}^{2}$, and rms spread $\gamma_{\mathrm{o}} \sigma$. The static wiggler magnetic field has period length $\lambda_{\mathrm{w}}$, wavenumber $k_{w}=2 \pi / \lambda_{w}$. The resonant radiation frequency $\omega_{r}=k_{r} c$ of the FEL is determined by $k_{r}=2 \gamma_{o}^{2} k_{w} /\left(1+K^{2}\right)$, where $K=e B_{r m s} / k_{w} m c$ and $B_{r m s}$ is the rms value of the wiggler magnetic field (mks units). We assume either a helical wiggler, or a flat wiggler with parabolic pole faces. After averaging over the fast wiggler oscillations, the transverse electron motion is 'described by harmonic betatron oscillations [11] in the transverse displacement $d^{2} \vec{R} / d z^{2}=-k_{\beta}^{2} \vec{R}$. In the absence of external focusing, the betatron wavenumber [11] $\mathrm{k}_{\beta}=\mathrm{Kk}_{\mathrm{w}} /(\gamma / 2)$. Natural focusing does not cause longitudinal velocity modulation. For external focusing, we ignore the longitudinal velocity modulation.

Initially, we assume the electron beam has a uniform longitudinal density, and a uniform "waterbag" distribution inside a four-dimensional sphere in the four-dimensional transverse phase space $\vec{R}=(x, y), \vec{R}^{\prime}=d \vec{R} / d z=\left(x^{\prime}, y^{\prime}\right)$ :

$$
U\left(\vec{R}, \vec{R}^{\prime}\right)=\frac{n_{o}}{\pi k_{\beta}^{2} R_{o}^{2}} \theta\left(k_{\beta}^{2} R_{o}^{2}-k_{\beta}^{2} R^{2}-R^{2}\right),
$$

where the step function $\theta(v)=1$ for $v>0$ and $\theta(v)=0$ for $v<0$. Integrating $U\left(\bar{R}, \bar{R}^{\prime}\right)$ over the angular deviation $\overrightarrow{\mathrm{R}}^{\prime}$, one obtains the parabolic transverse density profile:

$g(\vec{R})=n_{0}\left(1-R^{2} / R_{0}^{2}\right)$ for $R<R_{0}$, and $g(\vec{R})=0$ for $R>R_{0}$. The peak electron density is $n_{0}$, and the electron beam current is $I_{0}=e_{0} c \pi R_{o}^{2} / 2$. The rms transverse emittance $\epsilon$ of the matched electron beam is defined by

$$
E=\left(\left\langle x^{2}\right\rangle\left\langle x^{2}\right\rangle\right)^{1 / 2}=\left(\left\langle y^{2}\right\rangle\left\langle y^{2}\right\rangle\right)^{1 / 2}=k_{\beta} R_{0}^{2} / 6
$$


We consider the linear region before saturation, and write the electric field of the fundamental guided laser mode of frequency $\omega$ in the form:

$$
E(\vec{R}, \omega) e^{-i \mu k_{w}^{2}} e^{-i \omega(t-y / c)} \hat{e}+c . c .
$$

where $\hat{\mathrm{e}}$ is either the helical polarization vector $(\hat{\mathrm{x}}+\mathrm{i} \hat{\mathrm{y}}) / \sqrt{2}$, or the linear polarization vector $\widehat{\chi}$. The function $E(\vec{R}, \omega)$ describes the transverse mode profile. The factor $\exp \left(-\mathrm{i} \mu \mathrm{k}_{\mathrm{w}} \mathrm{z}\right)$ describes the deviation from free space propagation and $2 \pi \operatorname{Im} \mu$ is the growth rate per wiggler period. The e-folding length $L_{G}$ of the power of the amplified mode is given by

$$
\frac{1}{2 L_{G}}=k_{w} I m \mu
$$

Assuming $\gamma_{0}>>1$, so space charge effects are negligible, Vlasov-Maxwell equations have been used to derive a dispersion relation [2] determining $\mu$ and $E(\vec{R})$. From this dispersion relation, we have shown [1] that the e-folding length $\mathrm{L}_{\mathrm{O}}$ of the amplified power can be expressed in the scaled form:

$$
\frac{1}{2 k_{w} L_{G}}=\operatorname{Im} \mu=D G\left(k_{r} \in, \frac{\sigma}{D}, \frac{k_{\beta}}{k_{w} D}, \frac{\omega-\omega_{r}}{\omega_{r} D}\right) .
$$

The scaling parameter $\mathrm{D}$ is a measure of the transverse electron current,

$$
D=\left(\frac{4 e Z_{0}}{\pi m c^{2}} \frac{K^{2}}{1+K^{2}} \frac{I_{0}}{\gamma_{0}}\right)^{1 / 2}[J J]
$$

where $Z_{o}=377 \Omega$ is the impedance of free space and $[\mathrm{JJ}]=1$ for a helical wiggler and for a flat wiggler, 


$$
[J]=J_{0}\left(K^{2} / 2\left(1+K^{2}\right)\right)-J_{1}\left(K^{2} / 2\left(1+K^{2}\right)\right)
$$

Utilizing the variational approximation, we have determined [1] the universal gain function $\mathrm{G}=\operatorname{Im} \mu / \mathrm{D}$. Because of the scaling law, the entire physical parameter space can be described by the dependence of $G$ on four dimensionless scaled variables. In Figs $1 \mathrm{a}, \mathrm{b}, \mathrm{c}$ we plot $\mathrm{G}=\operatorname{Im} \mu / \mathrm{D}$ against $2 \mathrm{k}_{\mathrm{r}} \epsilon=4 \pi \epsilon / \lambda_{\mathrm{r}}$, for several values of the scaled focusing strength $\mathrm{k}_{\beta} /\left(\mathrm{k}_{\mathrm{w}} \mathrm{D}\right)$, corresponding to scaled energy spread (a) $\sigma / \mathrm{D}=0$, (b) $\sigma / \mathrm{D}=0.1$, (c) $\sigma / \mathrm{D}=0.2$. For each point $\left(2 \mathrm{k}_{\mathrm{T}} \epsilon, \mathrm{k}_{\beta} /\left(\mathrm{k}_{\mathrm{w}} \mathrm{D}\right), \sigma / \mathrm{D}\right)$, the scaled detuning is chosen to be

$$
\frac{\omega-\omega_{r}}{\omega_{r} D}=-3\left(\frac{k_{\beta}}{k_{w} D}\right) k_{r} \epsilon
$$

which we have found to yield near maximum gain. This detuning corresponds to the reduction of the average longitudinal velocity due to the nonvanishing emittance and focusing by the wiggler. Figs. 1a,b,c cover most of the practical range of the FEL parameters, and they can be used to obtain quick estimate; of the e-folding length.

To check the accuracy of our results, we have compared our calculation with several simulation codes $[7,8,9]$. In Table 1 , we compare our calculation with FELEX, as calculated by J. Goldstein [12]. The parameters for case 1 are the following: $\gamma_{o}=362$, normalized rms emittance $\epsilon_{\mathrm{n}}=7.74 \mathrm{~mm}$-mrad, rms relative energy spread $\sigma=6.6 \times 10^{-4}$, current $\mathrm{I}=300$ Amp, the peak on-axis magnetic field $B_{w}=0.75$ Tesla, wiggler period $\lambda_{\mathrm{w}}=1.6 \mathrm{~cm}$. The natural betatron wavelength for these parameters is $\lambda_{\beta}=10.3 \mathrm{~m}$, and the matched edge electron beam size is $0.459 \mathrm{~mm}$. The radiation wavelength is $\lambda=995.4 \AA$. Our result for power gain length is $\mathrm{L}_{\mathrm{O}}=108 \mathrm{~cm}$, as compared to $105 \mathrm{~cm}$, given by J. Goldstein. The case 2 is different from case 1 in that the normalized emittance is doubled, and correspondingly the gain length is 
Increased to $271 \mathrm{~cm}$, as compared to the simulation value $268 \mathrm{~cm}$. The case 3 is obtained from case 1 by doubling the energy spread, and the gain length is $164 \mathrm{~cm}$, as compared to the simulation value $166 \mathrm{~cm}$. In cases 4 and 5 , the betatron wavelength has been decreased by a factor of 2 and 3 respectively, relative to the case 1 , and for both cases the gain lengths only change slightly. Table 1 shows our results agree with simulation to within a few percent.

In Fig. 2, we compare the power gain length for different detuning from case 1 . The simulation used the code TDA as provided by J. Wurtele from MIT [9]. For small deviation from the minimum gain length, the agreement is within a few percent. For very large detuning from the minimum gain length, it becomes difficult to determine the gain length by simulation, because it is difficult to identify the domination of a single mode. The errors become larger. The figure also shows that Eq. (7) accurately gives the detuning for minimum gain length.

An alternate form of the scaling relation is:

$$
\frac{1}{2 k_{w} L_{G}}=\operatorname{Im} \mu=\rho F\left(\tilde{a}, \frac{\sigma}{\rho}, \frac{k_{\beta}}{k_{w} \rho}, \frac{\omega-\omega_{r}}{\omega_{r} \rho}\right),
$$

which follows from Eq. (6) upon noting that $D=2 \rho \bar{a}$, where $\rho$ is the Pierce parameter $[13,1]$ corresponding to the peak density $n_{0}$, and the scaled electron beam radius $a$ is defined by [14],

$$
\ddot{a}^{2}=2 \rho\left(2 k_{w} k_{r} R_{o}^{2}\right)=24\left(k_{r} \in\right)\left(\rho k_{w} / k_{\beta}\right) .
$$

Up to a multiplicative constant $\mathrm{a}^{2} \propto \mathrm{k}_{\mathrm{r}} \mathrm{R}_{\mathrm{o}}{ }^{2} / \mathrm{L}_{\mathrm{o}}{ }^{\mathrm{l}}$, the ratio of the Rayleigh range to the onedimensional gain length $\mathrm{L}_{\mathrm{g}}{ }^{1 \mathrm{D}} \propto\left(2 \rho \mathrm{k}_{\mathrm{w}}\right)^{-1}$.

For a parallel electri $n$ beam with parabolic transverse density profile: 
$g_{0}(R)=n_{0}\left(1-R^{2} / R_{0}{ }^{2}\right)$ for $R<R_{0}$ and $g_{0}(R)=0$ for $R>R_{0}$, we have obtained [10] exact results as a function of electron beam size and energy spread (Gaussian distribution). We have compared the variational approximation for the universal scaling function of Eq. (12) with the exact results. In Fig. 3, we plot the scaled growth rate $\operatorname{Im} \mu / 2 \rho$ versus the scaled energy spread $\sigma / 2 \rho$, for the scaled electron beam radius $\overline{\mathrm{a}}=1$ and zero detuning $\omega=\omega_{\mathrm{r}}$. The dashed line is the exact solution derived in ref. [10] using confluent hypergeometric functions. The solid line is calculated from the variational approximation. The agreement is good to about $1 \%$. The same good agreement holds for the full range from large to small beam size ( $\bar{a}>>1$ to $\mathrm{a}<<1)$.

The limit of a parallel beam corresponds to $\mathrm{k}_{\beta} \rightarrow 0, \epsilon \rightarrow 0$ with $\epsilon / \mathrm{k}_{\beta}=\mathrm{R}_{\mathrm{o}}{ }^{2} / 6$ finite. The gain length goes over to the one-dimensional value for a large.

\section{Acknowledgements}

We have benefitted from discussions with R. Palmer and C. Pellegrini. We thank J.C. Goldstein for provir'ing some results from the simulation code FELEX and E.T. Scharlemann for providing results from FRED. We also want to express our appreciation to J. Wurtele for kindly providing the simulation code TDA, which has allowed us to make a detailed comparison between our theory and simulation.

\section{DISCLAIMER}

This report was prepared as an account of work sponsored by an agency of the United States Government. Neither the United States Government nor any agency thereof, nor any of their employees, makes any warranty, express or implied, or assumes any legal liability or responsibility for the accuracy, completeness, or usefulness of any information, apparatus, product, or process disclosed, or represents that its use would not infringe privately owned rights. Refer. ence herein to any specific commercial product, process, or service by tride name, lrademark, manufacturer, or otherwise does not necessarily constitute or imply its endorsement, recommendation, or favoring by the United States Government or any agency thereof. The views and opinions of authors expressed herein do not necessarily state or reflect those of the United States Government or any agency thereof. 


\section{References}

1. L.H. Yu, S. Krinsky and R.L. Gluckstern, Phys. Rev. Lett. 64, 3011 (1990).

2. L.H. Yu and S. Krinsky, Phys. Lett. A129, 463 (1988).

3. A dispersion relation of this type wals considered by K.J. Kim, Phys. Rev. Lett. 57, 1871 (1986).

4. M. Xie and D.A.G. Deacon, Nucl. Instrum. Methods A250, 426 (1986).

5. C. Pellegrini, Nucl. Instrum. Methods A272, 364 (1988).

6. W.A. Barletta and A.M. Sessler, in Proc. INFN International School on Electromagnetic Radiation and Particle Beam Acceleration, Varenna, Italy (1988).

7. J.C. Goldstein, T.F. Wang, B.E. Newnam and B.D. McVey, Proc. 1987 Particle Accelerator Conference, Washington, D.C., p. 202.

8. E.T. Scharlemann and W.M. Fawley, Proc. SPIE $\underline{642}, 2$ (1986).

9. T.M. Tran and J.S. Wurtele, Computer Phys. Comm. 54, 263 (1989).

10. S. Krinsky, L.H. Yu and R.L. Gluckstern, unpublished.

11. E.T. Scharlemann, J. Appl. Phys. 58, 2154 (1985).

12. J.C. Goldstein, private communication.

13. R. Bonifacio, C. Pellegrini and L.M. Narducci, Opt. Commun. 50, 373 (1984).

14. S. Krinsky and L.H. Yu, Phys. Rev, A $\underline{35}, 3406$ (1987). 
Table 1. Gain Lengths Calculated by Simulation and Solving Dispersion Relation

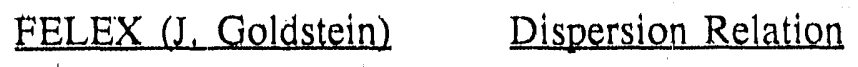

$\begin{array}{lll}\text { Case 1 } & 105 \mathrm{~cm} & 108 \mathrm{~cm} \\ \text { Case 2 }\left(\epsilon_{\mathrm{n}} \times 2\right) & 268 \mathrm{~cm} & 271 \mathrm{~cm} \\ \text { Case 3 }(\sigma \times 2) & 166 \mathrm{~cm} & 164 \mathrm{~cm} \\ \text { Case } 4\left(\lambda_{\beta} / 2\right) & 107 \mathrm{~cm} & 104 \mathrm{~cm} \\ \text { Case } 5\left(\lambda_{\beta} / 3\right) & 115 \mathrm{~cm} & 108 \mathrm{~cm}\end{array}$




\section{Figure Captions}

Fig. 1.

Scaling function vs. scaled emittance for several values of $k_{\beta} /\left(k_{w} D\right)$, corresponding to scaled energy spreads (a) $\sigma / D=0$, (b) $\sigma / D=0.1$, (c) $\sigma / D=$ 0.2, with the detuning as given in Eq. (7).

Fig. 2 . Comparision of power gain lengths calculated from dispersion relation and from the TDA simulation code for the case 1 of Table 1, as a function of detuning.

Fig. 3. Comparison of scaled growth rate $\operatorname{Im} \mu / 2 \rho$ calculated from the variational approximation (solid curve) and from the exact solution (dashed curve). 


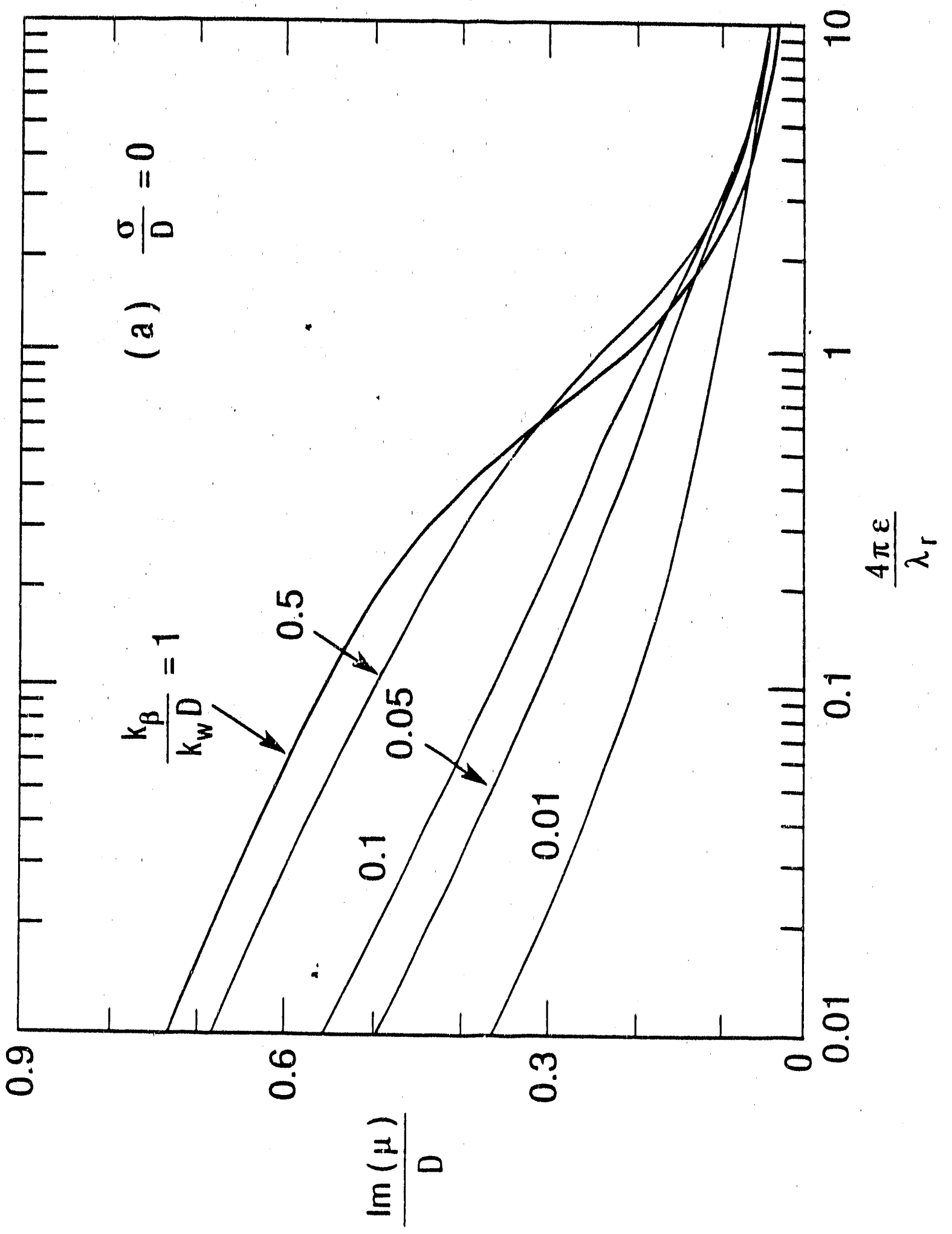




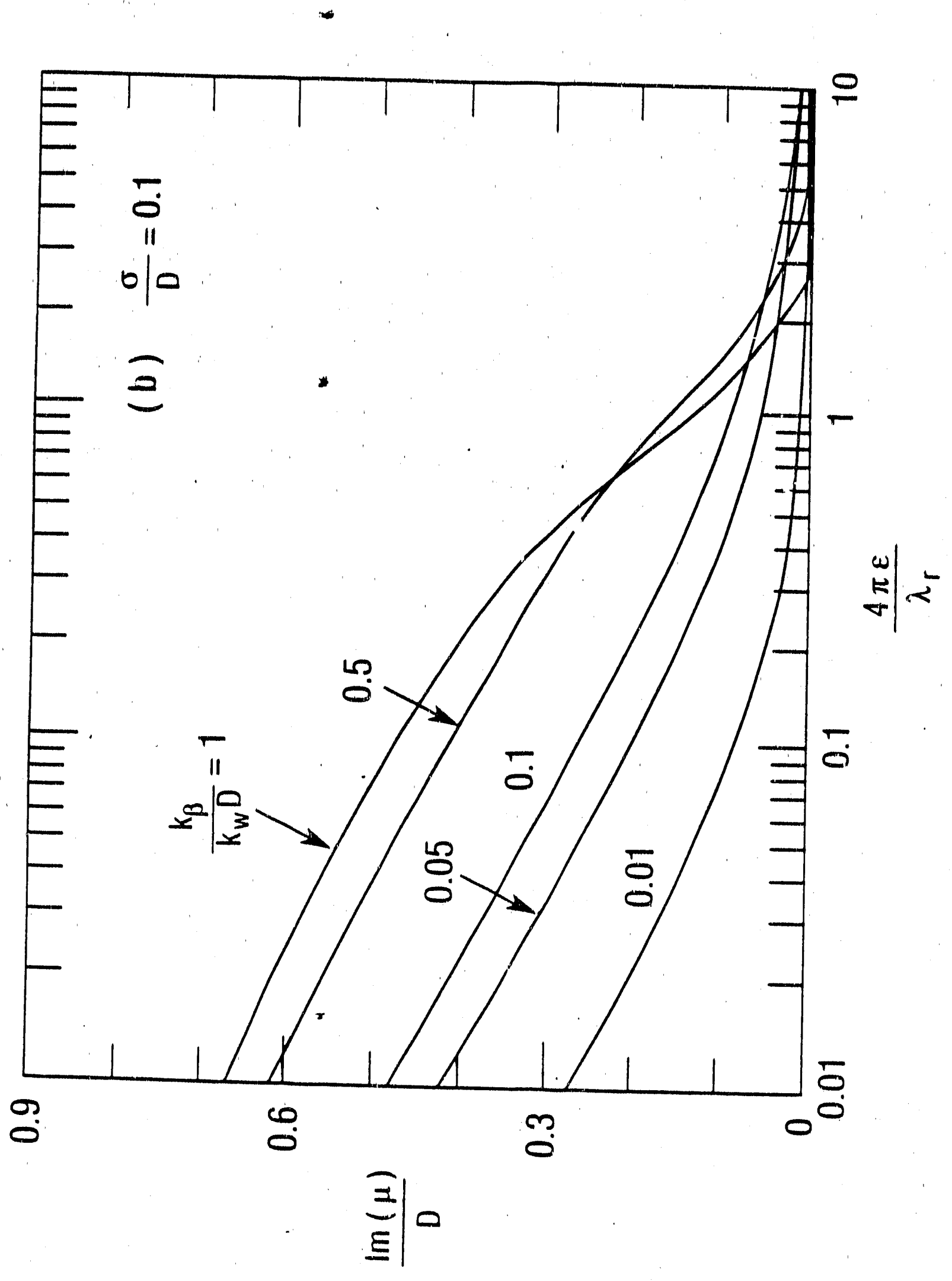




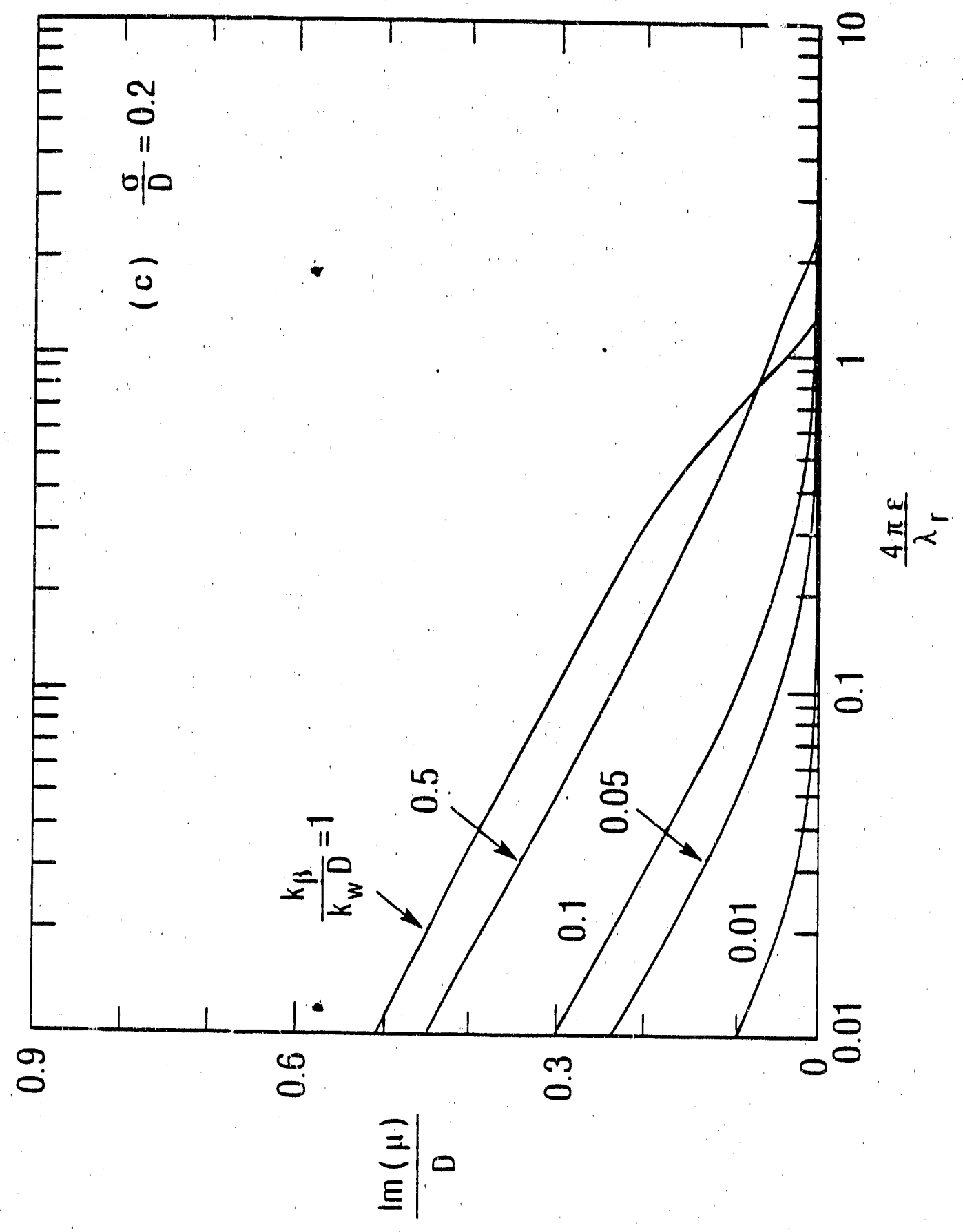




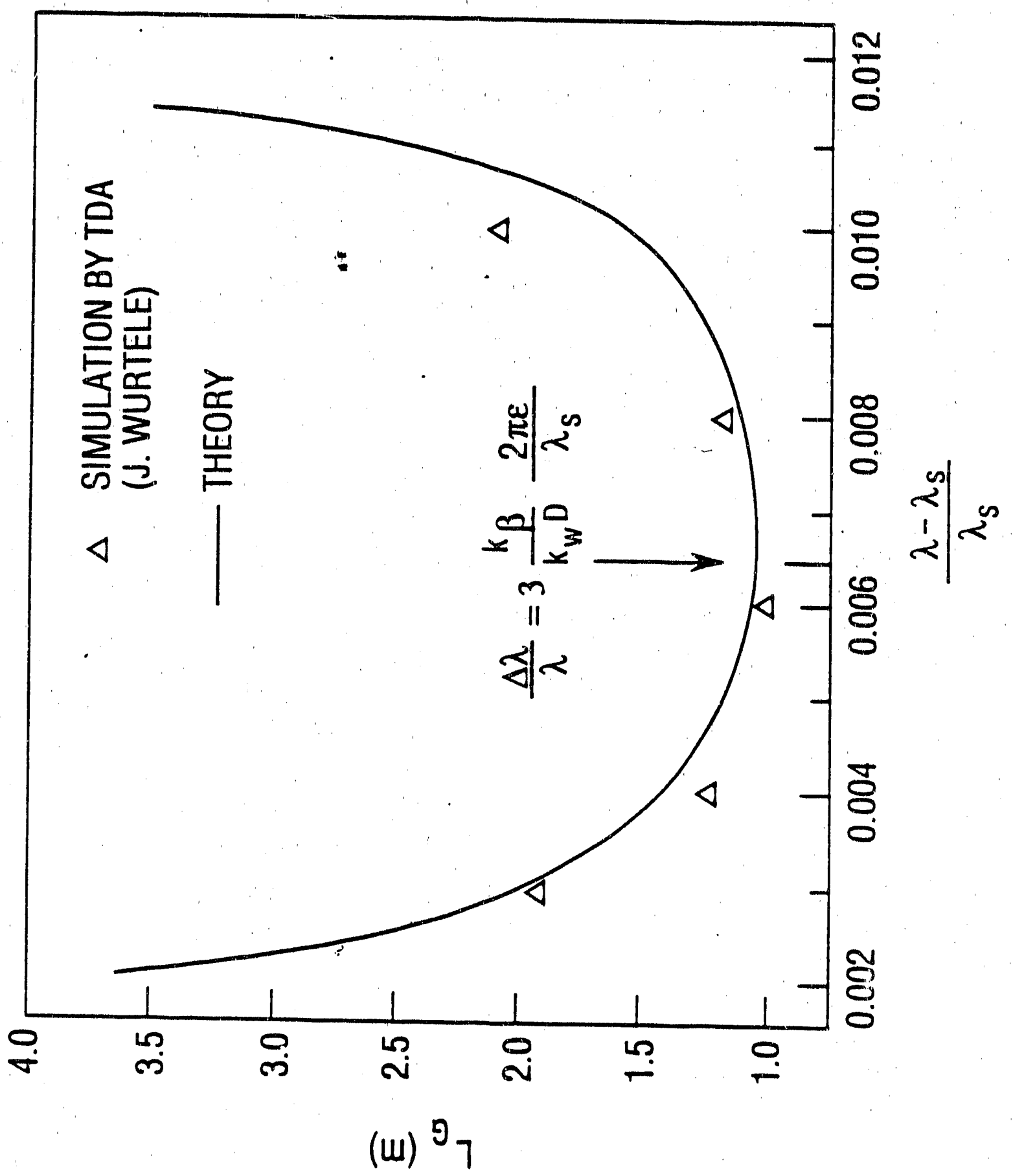




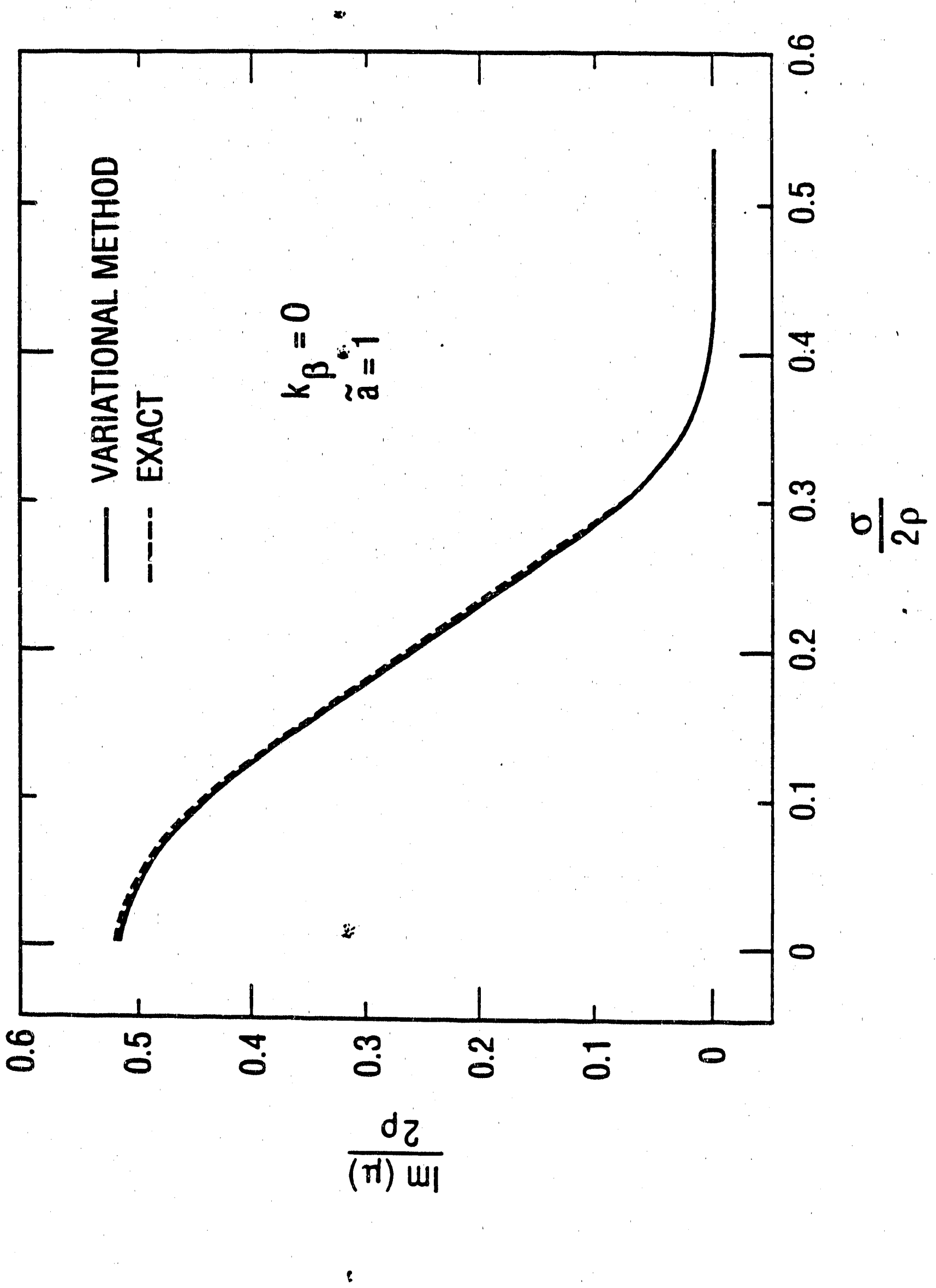



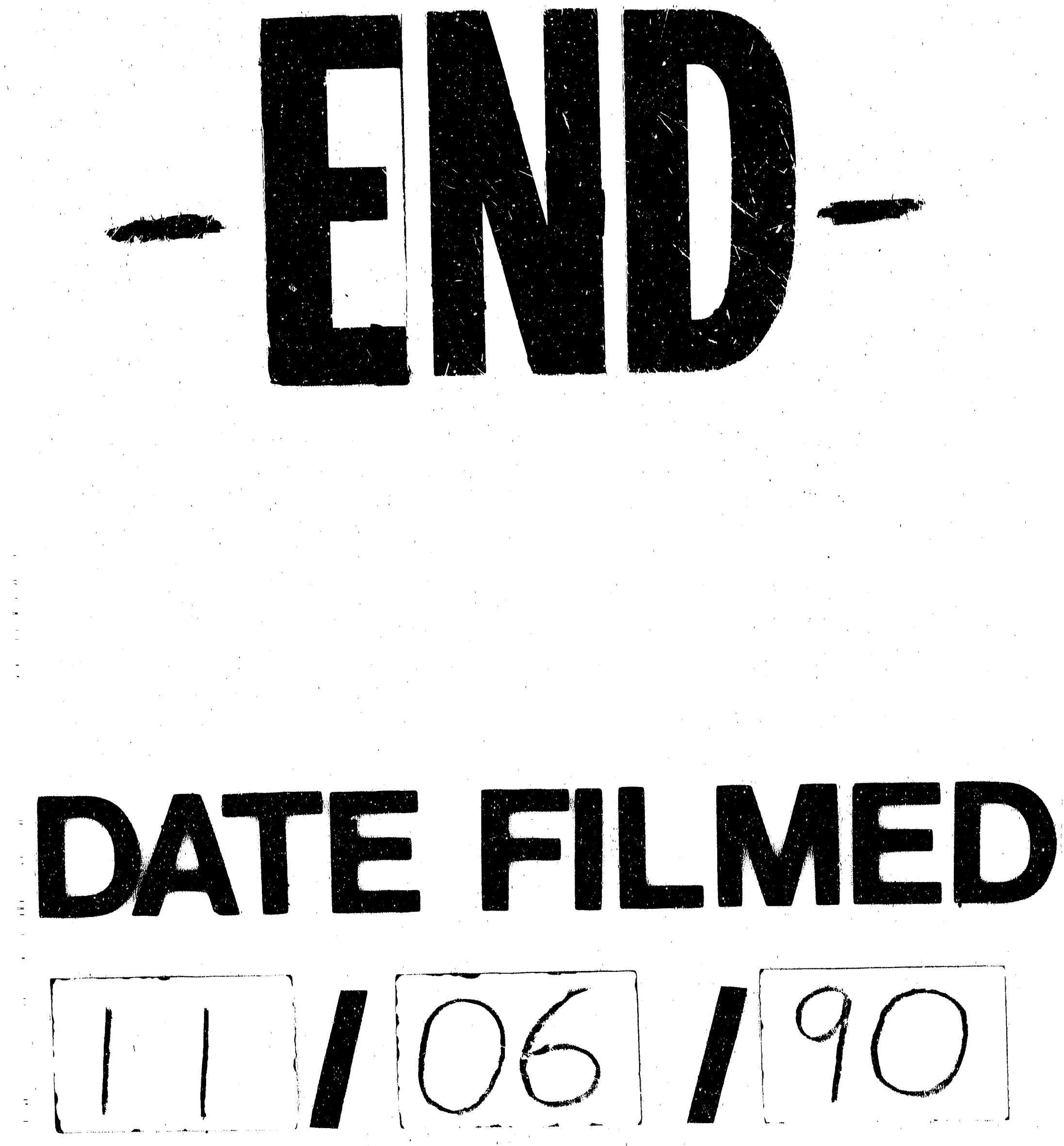
